\title{
Current-Induced Magnetic Dynamics in Asymmetric Ferromagnetic Single-Electron Devices
}

\author{
M. KOWALIK \\ Institute of Physics, Adam Mickiewicz University \\ Umultowska 85, 61-614 Poznań, Poland
}

\begin{abstract}
The theoretical calculations of possible dynamics for a localized magnetic moment of the central electrode in ferromagnetic single-electron devices are presented. The spin-transfer torque from spin current absorbed by the central electrode is calculated. Thereafter, an in-plane component of spin torque is regarded during the integration of the Landau-Lifshitz-Gilbert equation and the time evolution of the localized magnetic moment of the central electrode is obtained. The necessary conditions for switching of the magnetic moment are discussed.
\end{abstract}

PACS numbers: 72.25.-b, 73.23.Hk, 85.75.-d

\section{Introduction}

Theoretically predicted spin transfer torque $[1,2]$ which can lead to current-induced magnetic switching is possible to measure [3, 4]. Motivated by the recent theoretical and experimental results, the necessary conditions for current-induced magnetic switching in the case of an asymmetric ferromagnetic single-electron transistor (SET) are investigated.

The investigated model assumes only the existence of in-plane component of the spin torque. The charge and spin currents, and consequently also the spin torque, have been calculated in the sequential transport regime, from the relevant master equation. The spin torque has been calculated from the spin current absorbed by the central electrode. The Landau-Lifshitz-Gilbert (LLG) equation, with the spin torque included, has been then used to study current-induced switching and dynamics.

In Sect. 2 the analyzed model is presented. The method used for the theoretical description of the spin-dependent transport and thus, current-induced torque, as well as the time evolution equation for the net spin moment of the island, are introduced. Numerical results and discussion are presented in Sect. 3. Finally, the conclusions are given in Sect. 4.

\section{Model and method}

The considered asymmetric SET consists of a small ferromagnetic central electrode, which is connected by tunnel barriers to two external ferromagnetic electrodes, to which a transport voltage is applied. Apart from this, an external gate voltage $V_{\mathrm{g}}$ can be applied capacitively (with the corresponding capacitance $C_{\mathrm{g}}$ ) to the island (see Fig. 1). That model was already presented elsewhere [5].
At the beginning, spin moments of the leads and the island are oriented in a common plane. The net spin moments of the left and right leads can be aligned both parallel and antiparallel, whereas the net spin moment of the island can be oriented in arbitrary direction.

The tunnel barriers (tunnel junctions) are characterized by the capacitance $C_{j}$, and barrier resistances $R_{j}^{P, \sigma}$, $R_{j}^{A P, \sigma}$, for majority $(\sigma=+)$ and minority $(\sigma=-)$ electrons, where configuration is parallel $(\mathrm{P})$ and antiparallel (AP), in the case of the left $(j=l)$ and right $(j=r)$ electrode, respectively.

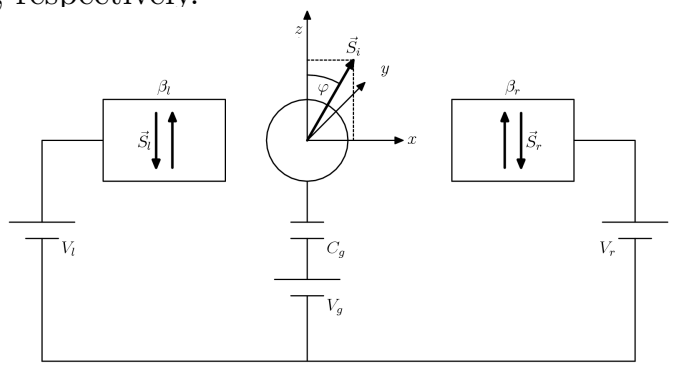

Fig. 1. A schematic diagram of the asymmetric ferromagnetic SET. The vectors $\boldsymbol{S}_{\mathrm{l}}, \boldsymbol{S}_{\mathrm{r}}$ and $\boldsymbol{S}_{\mathrm{i}}$ are the unit vectors along the classical net spin moments of the left and right electrodes and of the island, respectively. Potential applied to the left and right electrode are $V_{1}$ and $V_{\mathrm{r}}$, where the island is connected capacitively to the gate voltage $V_{g}$.

The island is assumed to be a sphere of the radius $2 \mathrm{~nm}$. Therefore, we can consider its energy spectrum as the continuous one. Nevertheless, the corresponding charging energy associated with addition of one extra electron charge on the island may still be larger than the thermal energy. Moreover, the barrier resistances are assumed to be much larger than the quantum resistance $R_{q}=h / e^{2}$. 
Following the above assumptions, we restrict our calculations to the sequential tunneling regime, and apply the so-called orthodox tunneling theory [6] based on the master equation and the Fermi golden rule. The spin splitting of the Fermi level for a given value of the spin relaxation time is calculated self-consistently from the following spin balance equations [7]:

$$
\frac{1}{e}\left(I_{1}{ }^{\sigma}-I_{\mathrm{r}}{ }^{\sigma}\right)-\frac{D_{i} \Omega_{i}}{\tau_{s f}} \Delta E^{\sigma}=0,
$$

where $\sigma=+,-$ refers to the spin-majority and spin-minority electrons, respectively. $I_{1}^{\sigma}\left(I_{\mathrm{r}}^{\sigma}\right)$ is the current flowing in the $\sigma$ channel through the left (right) junction. $D_{i}$ denotes the density of states of the island, $\Omega_{i}$ is the island's volume, while $\tau_{s f}$ is the spin relaxation time.

Each of the electrode could be made of different ferromagnetic material, which is included via different values of a spin asymmetry coefficients $\beta_{\mathrm{l}}, \beta_{\mathrm{i}}$ and $\beta_{\mathrm{r}}$ for the left, central, and right electrode, respectively. The spin asymmetry coefficient for the given electrode $j$ is defined as $\beta_{j}=D^{+} / D^{-}$, where $D^{+}$denotes the density of states for the majority electrons, whereas density of states of minority electrons are given by $D^{-}$.

It can be easily shown, using that spin asymmetry coefficients, that the precise relationships for the resistances of the $j$-th barrier, are given as

$$
R_{j}^{\mathrm{P},-}=\beta_{j} \beta_{i} R_{j}^{\mathrm{P},+}, \quad R_{j}^{\mathrm{AP},+}=\sqrt{\frac{\beta_{j}}{\beta_{i}} R_{j}^{\mathrm{P},+} R_{j}^{\mathrm{P},-},}
$$

and

$$
R_{j}^{\mathrm{AP},-}=\sqrt{\frac{\beta_{i}}{\beta_{j}} R_{j}^{\mathrm{P},+} R_{j}^{\mathrm{P},-}},
$$

where $j=1$, r.

The analysis of the spin current absorbed by the island allows to express the in-plane component of the spin-transfer torque exerted on the island. The detailed formula for that torque was already presented elsewhere [5].

The time dependence of all three components of the net spin moment of the island $\boldsymbol{S}_{\mathrm{i}}$ are obtained by integrating of the generalized LLG equation. It means LLG equation with the additional term coming from the spin-current induced torque taken into account $\left(\boldsymbol{\tau}_{i}\right)$ [8], given as

$$
\frac{\mathrm{d} \boldsymbol{S}_{\mathrm{i}}}{\mathrm{d} t}=-\gamma_{\mathrm{g}} \mu_{0} \boldsymbol{S}_{\mathrm{i}} \times \boldsymbol{H}_{\mathrm{eff}}-\alpha \boldsymbol{S}_{\mathrm{i}} \times \frac{\mathrm{d} \boldsymbol{S}_{\mathrm{i}}}{\mathrm{d} t}+\frac{\gamma_{\mathrm{g}}}{M_{\mathrm{s}} V} \boldsymbol{\tau}_{i},
$$

where $\boldsymbol{S}_{\mathrm{i}}$ is the unit vector along the net spin moment of the island, $\gamma_{\mathrm{g}}$ - a value of the gyromagnetic ratio, $\mu_{0}$ is the magnetic vacuum permeability, $\alpha$ is a damping parameter, $\boldsymbol{H}_{\text {eff }}$ is an effective magnetic field acting on the central electrode, $M_{\mathrm{s}}$ is the saturation magnetization and $V$ is the volume of the island.

The effective field is given as

$$
\boldsymbol{H}_{\mathrm{eff}}=\boldsymbol{H}_{\mathrm{ext}}-H_{\mathrm{a}}\left(\boldsymbol{S}_{\mathrm{i}} \cdot \boldsymbol{z}\right) \boldsymbol{z}+\boldsymbol{H}_{\mathrm{d}} .
$$

$\boldsymbol{H}_{\text {ext }}$ is the external field, $H_{\mathrm{a}}$ is the magnetic anisotropy field, $\boldsymbol{H}_{\mathrm{d}}$ stands for the demagnetization field, and $\boldsymbol{z}$ is a unit vector along the $z$ direction.

\section{Numerical results and discussion}

The parallel and antiparallel configurations of the external electrodes were considered. It appeared that only in the antiparallel configurations (first when $\boldsymbol{S}_{1}$ pointed along the positive $z$-th direction and $\boldsymbol{S}_{\mathrm{r}}$ in the reverse direction, and the second just the opposite), the switching of the net spin moment of the island, using only the spin polarized current, is possible. The time dependence of the $z$-th component of the net spin moment of the central electrode for two opposite directions of the current flowing through the system are presented in Fig. 2. We can see that for the charge current flowing from left electrode to right, the net spin moment of the island $\boldsymbol{S}_{\mathrm{i}}$ aligned to the net spin moment of the right electrode $\boldsymbol{S}_{\mathrm{r}}$. If we reverse the direction of the charge current flowing through the system, it is just the opposite case, i.e. $\boldsymbol{S}_{\mathrm{i}}$ aligned to $\boldsymbol{S}_{\mathbf{l}}$. That switching occurs due to the fact that in-plane component of the current-induced torque should exhibit bias asymmetry [9], which is also the case for the considered system. The similar situation takes place for the second possible antiparallel configuration, where the net spin moment of the left electrode is assumed to point along to the negative $z$-th direction whereas the net spin moment of the right electrode in the opposite direction (see Fig. 3).

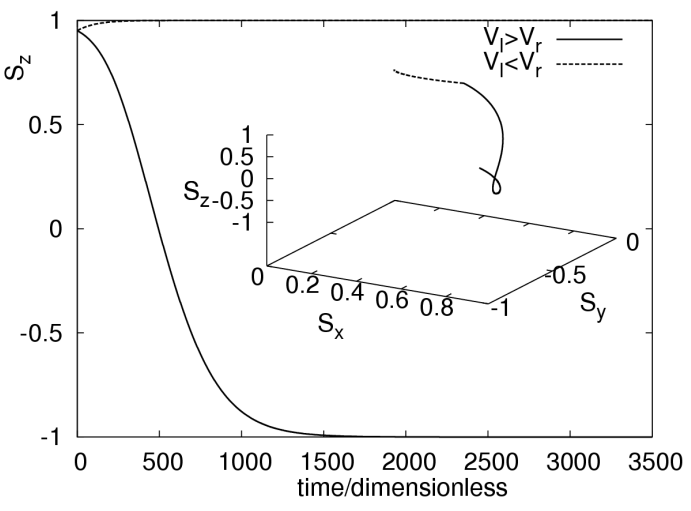

Fig. 2. The time dependence of the $z$-th component of the net spin moment of the island for charge current flow from the left electrode to right one $\left(V_{1}>V_{\mathrm{r}}\right)$ and for the charge current flow from right electrode to the left one $\left(V_{1}<V_{\mathrm{r}}\right)$. The net spin moment of the left electrode is assumed to point along to the positive $z$ th direction whereas the net spin moment of the right electrode in the opposite direction. The inset shows possible switching as a path in a coordinate system given by all three components of the net spin moment of the island.

If it is not stated explicitly, the parameters used in all calculations are as follows. The initial position of the net spin moment of the island is given by $\varphi=\pi / 10$ and $\eta=0$, where $\varphi$ and $\eta$ are the polar and the azimuthal angles, respectively, related with the coordinate system of the island. We considered the symmetrical applied voltage, so $\left|V_{1}\right|=\left|V_{\mathrm{r}}\right|=0.16 \mathrm{~V}$. For the presented data 


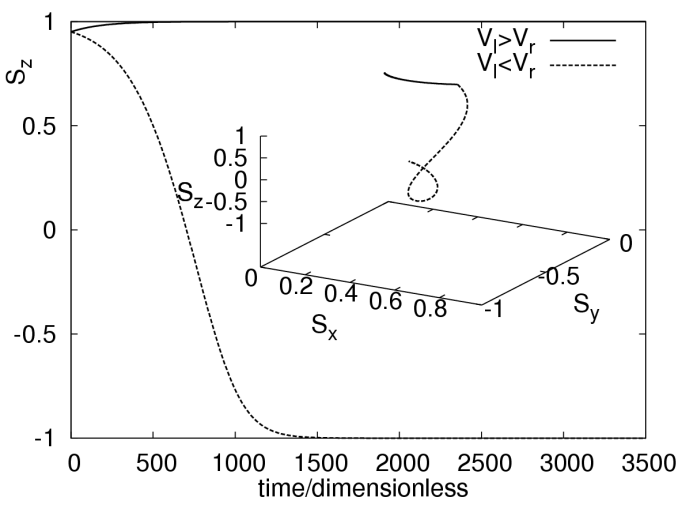

Fig. 3. The time dependence of the $z$-th component of the net spin moment of the island for charge current flow from the left electrode to right one $\left(V_{1}>V_{\mathrm{r}}\right)$ and for the charge current flow from right electrode to the left one $\left(V_{1}<V_{\mathrm{r}}\right)$. The net spin moment of the left electrode is assumed to point along to the negative $z$-th direction whereas the net spin moment of the right electrode in the opposite direction. The details concerning the values of parameters used for calculations are given in the text. The inset shows possible switching as a path in a coordinate system given by all three components of the net spin moment of the island.

we assume that all the electrodes are made of the same ferromagnetic material, given by $\beta_{1}=\beta_{\mathrm{i}}=\beta_{\mathrm{r}}=4$. The tunnel junctions are characterized by $C_{\mathrm{l}}=C_{\mathrm{r}}=1 \mathrm{aF}$ and $R_{1}^{\mathrm{P},+}=1 \mathrm{M} \Omega, R_{\mathrm{r}}^{\mathrm{P},+}=0.1 \mathrm{M} \Omega$. For the island we assumed: $M_{\mathrm{s}}=8 \times 10^{5} \mathrm{~A} / \mathrm{m}, H_{\mathrm{a}}=0.002 \times 10^{5} \mathrm{~A} / \mathrm{m}$, $\alpha=0.05, r=2 \mathrm{~nm}, D_{i} \Omega_{i}=1000 \mathrm{eV}^{-1}$ and $\tau_{s f}=10^{-6} \mathrm{~s}$. The temperature is $T=4.2 \mathrm{~K}$. For the gate we have chosen $V_{g}=0 \mathrm{~V}$ and $C_{g}=1 \mathrm{aF}$.

The switching time should be affected by the value of the applied voltage. According to achieved data (not shown), the noticeable enhancement of the switching time is possible just by increasing the current flow through the system. Moreover, it appeared that the switching time could be also changed by making the external electrodes from the different ferromagnetic materials.

\section{Conclusions}

The time evolution of the net spin moment of the central electrode $\boldsymbol{S}_{\mathrm{i}}$ of the asymmetric ferromagnetic SET was presented. The possibility of switching $\boldsymbol{S}_{\mathrm{i}}$ without any external magnetic field applied were investigated. It was shown that for antiparallel configurations of the net spin moments of the external electrodes, the switching can occur due to spin-transfer torque. Nevertheless, only in-plane component of spin-transfer torque were considered.

\section{Acknowledgments}

This work was supported by funds from the Ministry of Science and Higher Education as a research project in years 2006-2009.

\section{References}

[1] J.C. Slonczewski, J. Magn. Magn. Mater. 159, L1 (1996).

[2] L. Berger, Phys. Rev. B 54, 9353 (1996).

[3] Y. Huai, F. Albert, P. Nguyen, M. Pakala, T. Valet, Appl. Phys. Lett. 84, 3118 (2004).

[4] H. Meng, J. Wang, J.-P. Wang, Appl. Phys. Lett. 88, 082504 (2006).

[5] M. Kowalik, I. Weymann, J. Barnaś, Mater. Sci. Poland 25, 453 (2007).

[6] Single Charge Tunnelling, Eds. H. Grabert, M.H. Devoret, NATO Advanced Study Institute, Series B, Vol. 294, Plenum Press, New York 1992; Appl. Phys. Lett. 88, 082504 (2006).

[7] J. Barnaś, A. Fert, J. Magn. Magn. Mater. 192, 391 (1999).

[8] M. Gmitra, J. Barnaś, Phys. Rev. Lett. 96, 207205 (2006).

[9] I. Theodonis, N. Kioussis, A. Kalitsov, M. Chshiev, W.H. Butler, Phys. Rev. Lett. 97, 297205 (2006). 